\title{
BMJ Open Impact of pre-eclampsia on the cardiovascular health of the offspring: a cohort study protocol
}

\author{
Zahra Hoodbhoy, ${ }^{1}$ Babar Sultan Hasan, ${ }^{1}$ Nuruddin Mohammed, ${ }^{2}$ \\ Devyani Chowdhury ${ }^{3}$
}

To cite: Hoodbhoy Z, Hasan BS, Mohammed N, et al. Impact of pre-eclampsia on the cardiovascular health of the offspring: a cohort study protocol. BMJ Open 2018;8:e024331. doi:10.1136/ bmjopen-2018-024331

- Prepublication history for this paper is available online. To view these files, please visit the journal online (http://dx.doi. org/10.1136/bmjopen-2018024331).

Received 22 May 2018 Revised 17 August 2018 Accepted 20 August 2018

\section{Check for updates}

\section{(c) Author(s) (or their} employer(s)) 2018. Re-use permitted under CC BY-NC. No commercial re-use. See rights and permissions. Published by BMJ.

${ }^{1}$ Department of Paediatrics and Child Health, The Aga Khan University, Karachi, Pakistan 2Department of Obstetrics and Gynaecology, The Aga Khan University, Karachi, Pakistan ${ }^{3}$ Cardiology Care for Children, Philadelphia, Pennsylvania, USA

Correspondence to Dr Babar Sultan Hasan; babar.hasan@aku.edu

\section{ABSTRACT}

Introduction Pre-eclampsia is a common disorder associated with serious maternal and fetal complications. It is associated with abnormal placentation, which significantly reduces flow, resulting in a relative hypoxic state. These pathophysiological changes lead to subtle macrovascular and cardiac structural and functional changes in the fetus. This can predispose the child with maternal history of pre-eclampsia to risk of premature cardiovascular disease.

Methods and analysis The children will be identified from a cohort of women with pre-eclampsia. The study will be conducted at The Aga Khan University Hospital, Karachi. Inclusion criteria will be children who are between 2 and 5 years of age and have a maternal history of pre-eclampsia. The child's current weight, height and blood pressure will be recorded. A two-dimensional functional echocardiogram and vascular assessment will be performed to evaluate alterations in cardiac function as well as macrovascular remodelling in these children. Data will be presented as mean $\pm \mathrm{SD}$, median (IQR) or percentages as appropriate. Independent t-test or Mann-Whitney U test will be used for testing of continuous variables (based on the assumption of normality). $A p<0.05$ will be used to determine statistical significance.

Ethics and dissemination Ethical approval has been obtained from AKUH Ethics Review Committee. Findings will be disseminated through scientific publications and project summaries for the participants.

\section{INTRODUCTION}

Pre-eclampsia is a pregnancy-specific condition, defined by the sudden onset of hypertension (systolic blood pressure (BP) of $>140 \mathrm{~mm} \mathrm{Hg}$ and diastolic BP of $>90 \mathrm{~mm}$ $\mathrm{Hg}$, taken on two separate occasions at least 4-6hours apart) and significant proteinuria (excretion of $300 \mathrm{mg}$ or more of protein every 24 hours) after the 20th week of gestation. Hypertensive disorders of pregnancy account for $9 \%$ of maternal deaths in Asia and Africa. This translates into approximately 25000 maternal deaths in low/middle-income countries such as Asia and Africa as compared with only 150 maternal deaths in the industrialised world. ${ }^{2}$ This number may actually be higher

\section{Strengths and limitations of this study}

- This is one of the first studies to objectively assess cardiovascular risk in children (aged 2-5 years) born to pre-eclamptic mothers, including early-onset and late-onset pre-eclampsia.

- The aims of this study are supported by objective echocardiographic and vascular data.

- The current protocol does not include microvascular assessment.

- The cohort not controlled for management and outcome of pre-eclampsia.

once the revised American College of Obstetricians and Gynecologists guidelines on diagnosis of pre-eclampsia without proteinuria are incorporated. ${ }^{3}$

Pre-eclampsia may result in the development of recognised cardiovascular disease (CVD) risk factors in the offspring. A recent systematic review on GVD risk in children between the ages of 4 and 19 years stated that systolic BP was higher in children born to pre-eclamptic mothers as compared with controls (mean difference $=2.26 \mathrm{~mm} \mathrm{Hg}, 95 \% \mathrm{CI}$ 1.35 to 3.16 ), but this effect was not seen in diastolic BPs (mean difference $=1.48 \mathrm{~mm} \mathrm{Hg}$ ), $95 \%$ CI 0.82 to $2.14 .{ }^{4}$ It was also reported that children with a mean birth weight of $>2.5 \mathrm{~kg}$ with history of exposure to pre-eclampsia in utero, had a significantly increased body mass index $(\mathrm{BMI}) \quad\left(0.67 \mathrm{~kg} / \mathrm{m}^{2} ; 95 \%\right.$ CI 0.35 to $0.99 ; \mathrm{p}=0.0001)$ as compared with controls. ${ }^{4}$ These studies were limited by a lack of assessment of cardiac and vascular remodelling, alterations of which could have been present in this population predisposing the child to future CVD risk.

There are very few studies that have looked at cardiac function using echocardiography (ECHO) in children of pre-eclamptic mothers. CHASE study (cardiovascular health after pregnancy complications) conducted in children between the ages of 5 and 8 years born to 
mothers suffering from pre-eclampsia reported that these children had a shorter left ventricular (LV) end-diastolic length and increased late diastolic velocity ( $\mathrm{A}^{\prime}$ wave) that may be an indicator of diastolic dysfunction. ${ }^{5}$ However, the sample size of this study was very small $(n=25)$ and thus probably inadequately powered for any conclusive results.

There is limited literature on vascular function in children born to pre-eclamptic mothers. Aortic intima-media thickness (IMT) was significantly higher in neonates of mothers with pre-eclampsia (mean-0.36 $\pm 0.03 \mathrm{~mm}$ ) as compared with controls (mean-0.33 $\pm 0.03 \mathrm{~mm}$ ). ${ }^{6}$ This study was conducted in neonates and hence the effect of pre-eclampsia exposure later in childhood remained unexplored.

The clinical expression of pre-eclampsia can be further reclassified based on early-onset versus late-onset disease; early pre-eclampsia refers to the disease presenting at $<34$ weeks of gestation while late pre-eclampsia refers to manifestations $>34$ weeks. ${ }^{7}$ Lazdam et al have reported that children born to mothers with early-onset pre-eclampsia have significantly higher systolic BPs $(96.3 \pm 7.3 \mathrm{~mm} \mathrm{Hg})$ between 6 and 13 years of age as compared with those who were born to mothers with late onset of the disease $(88.4 \pm 7.6 \mathrm{~mm} \mathrm{Hg}) .{ }^{8}$ Despite this preliminary evidence, there is lack of literature on cardiac and vascular function in these subgroups.

The primary objective of this study is to do a detailed cardiovascular assessment by differences in BP, myocardial systolic, diastolic function, regional and global myocardial strain as well as changes in carotid IMT (cIMT) and pulse wave velocity (PWV) in children $2-5$ years of age born to pre-eclamptic as compared with normotensive mothers. The secondary objective of this study is to assess cardiac and vascular function in children 2-5 years of age born to early versus late pre-eclamptic mothers.

\section{METHODS AND ANALYSIS \\ Setting}

The study will be conducted at The Aga Khan University Hospital (AKUH), Karachi, Pakistan.

\section{Design}

This will be a prospective cohort study design with the exposure status of the child (ie, pre-eclampsia) being identified from maternal records at AKUH. All echocardiographic and vascular assessments to determine the cardiovascular risk of the exposed cohort will be performed during the course of the study. No such data are available in medical records. The study recruitment will be over an 18-month period.

\section{Inclusion and exclusion criteria}

The inclusion criteria for exposed group are children between 2 and 5 years of age at the time of enrolment in the study with history of pre-eclampsia in the mother during pregnancy as diagnosed by the obstetrician. The inclusion criteria for non-exposed group are children between 2 and 5 years of age born to mothers with a normal BP. The exclusion criteria for both groups are presence of maternal comorbids such as gestational hypertension, diabetes, maternal obesity (BMI $>25 \mathrm{~kg}$ / $\mathrm{m}^{2}$ ), hypothyroidism; chromosomal abnormalities and congenital heart disease. Inability to acquire all echocardiographic and at least one cIMT image, will also lead to exclusion of that patient from the analysis.

\section{Sample size calculation}

Differences in ratio of pulse wave Doppler (PWD) derived early diastolic filling velocity of left ventricle (E) to tissue Doppler imaging (TDI) derived early diastolic myocardial velocity (E') acquired at the base of the ventricular septum and TDI derived peak systolic myocardial velocity of the right ventricle $\left(\mathrm{S}^{\prime}\right.$ in $\mathrm{cm} / \mathrm{s}$ ) were used to calculate sample size as they have a high sensitivity to detect preclinical cardiac dysfunction in children. ${ }^{9}$ The sample size was calculated using WHO sample size calculator V.2.0 with $90 \%$ statistical power and using $5 \%$ level of significance. Published data of mean pulsed wave E' obtained in pre-eclamptic children $(2.18 \pm 0.33 \mathrm{~mm} / \mathrm{s})$ and controls $(2.02 \pm 0.31 \mathrm{~mm} / \mathrm{s})$ was used. ${ }^{5}$ Based on this data, a sample size of 80 in each group was calculated. A sample size of 22 in each group was calculated using similar assumptions for cIMT along with published data in pre-eclamptic children $(0.36 \pm 0.03 \mathrm{~mm})$ and controls $(0.33 \pm 0.03 \mathrm{~mm}){ }^{6}$ Based on these, we will recruit 80 children born to pre-eclamptic mothers and controls, respectively, with a minimum of 20 children in each of the early-onset versus late-onset disease group.

\section{Recruitment procedure}

Data for children who meet the inclusion criteria will be obtained from AKUH's Hospital Information Management System. Parents of these children would then be called by a trained data collector and briefed regarding the purpose of the study. For those who agree on the follow-up call, appointment for a two-dimensional functional echocardiographic study and vascular imaging of the child will be scheduled with a paediatric cardiologist in the morning.

The child's height, weight and BP will be recorded at the ECHO laboratory at AKUH. A focused ECHO will then be performed by a trained technician to assess cardiac function as well as cIMT and PWV. The procedure will take 15-20 min for each participant. All studies will be reviewed by two independent paediatric cardiologists (BSH and DC) who will be blinded to the patient exposure group. The findings of the echocardiogram will be shared with the parents after the ECHO is complete.

\section{Measures of outcomes}

Anthropometry

Weight and height of the child will be recorded using a weighing scale with a stadiometer (Detecto Scales WS\#149).

\section{BP measurement}

Resting BP of the child in right upper extremity will be recorded manually $3-5$ min apart. BP will be measured 


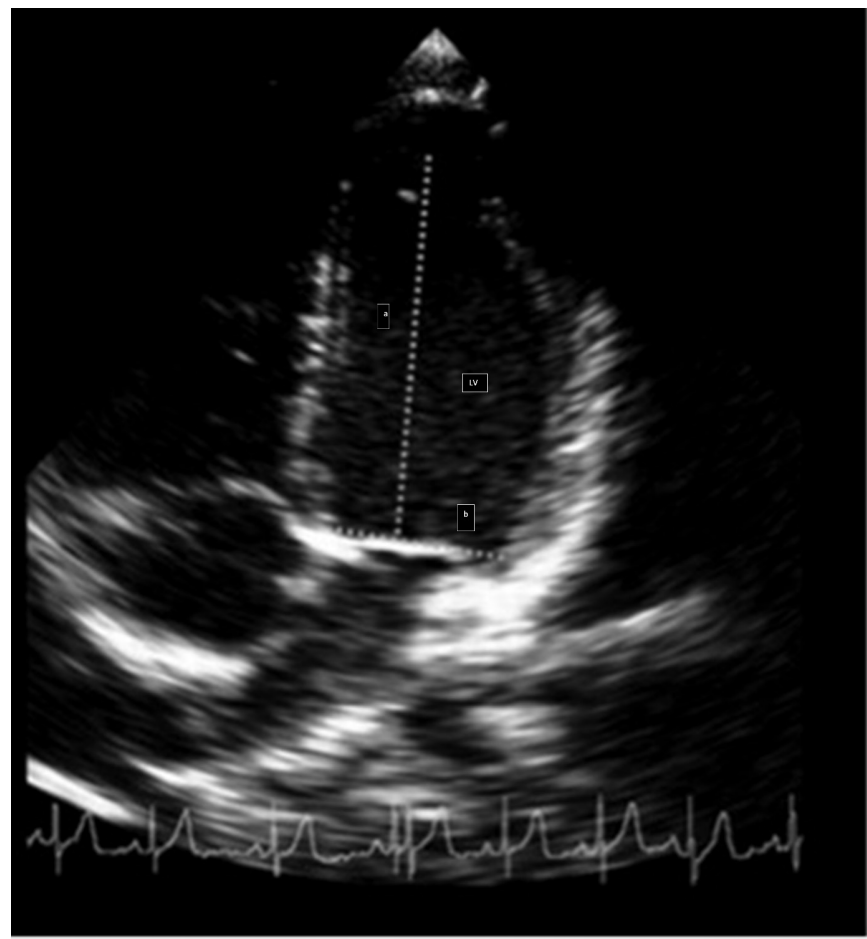

Figure 1 Measurement of sphericity index (in apical fourchamber view) (base to apex length/basal diameter of the ventricle).

manually with a sphygmomanometer with the appropriate cuff size. The average reading would be compared with the $\mathrm{z}$ scores available on a free software. ${ }^{10}$

\section{Echocardiographic examination}

All echocardiographic examinations will be performed using the GE Healthcare Vivid E9 (GE Healthcare, Waukesha, Wisconsin, USA) using 5-12 $\mathrm{MHz}$ phased array transducers. All cardiac parameters will be measured over three consecutive cardiac cycles and the average reading will be reported. The following parameters will be analysed:

\section{Cardiac morphometry}

This includes left atrium (LA) area, LV mass, sphericity index and relative wall thickness. LA area will be traced in the apical four-chamber view of the heart. Semiautomated tracing of the LA endocardium will then be performed to obtain LA area. These will be indexed to body surface area. ${ }^{11} \mathrm{LV}$ mass will be assessed using the $5 / 6$ $\mathrm{X}$ area $\mathrm{X}$ length method ${ }^{12}$ and $\mathrm{z}$ scores will be obtained. Linear measurements of base-to-apex length and basal diameter of left and right ventricles will be determined from an apical four-chamber view at end diastole. Sphericity index (base to apex length/basal diameter of the ventricle) for both the ventricles will then be calculated ${ }^{13}$ (refer to figure 1). LV end-diastolic septal and posterior wall thicknesses will be measured in parasternal short axis view. Relative wall thickness will be calculated as posterior +septal wall thickness/LV end-diastolic diameter ${ }^{14}$ (refer to figure 2).

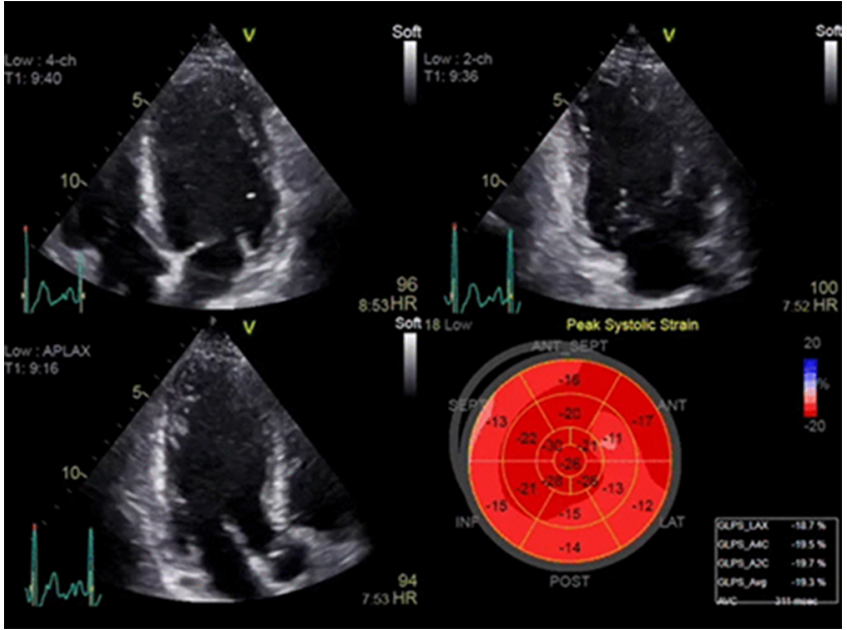

Figure 2 Global longitudinal strain from two, three and fourchamber view and bull's eye measurement of regional strain.

\section{Systolic function}

Measurements for LV includes ejection fraction, shortening fraction $(\mathrm{SF})$, stroke volume, heart rate, cardiac outputs (measured in parasternal long and short axis view) (assessed using the $5 / 6 \mathrm{X}$ area $\mathrm{X}$ length method) and mitral annular plane systolic excursion (measured by M-mode in apical four-chamber view).$^{15}$ Velocity of circumferential fibre contraction (VCFc) is a preload-independent measure of $\mathrm{LV}$ function and is inversely related to end-systolic wall stress (ESWS) ${ }^{16}$ The VCFc to ESWS relation is a preload independent, afterload incorporated function of contractility. ${ }^{16}$ It is calculated using the formula $\mathrm{SF} /$ heart rate adjusted ejection time. For $\mathrm{SF}$, we will be using the formula end-diastolic diameter - end-systolic diameter/end-diastolic diameter. ESWS will be calculated by using Grossman's formula (1.35 (LV end-systolic pressure) (LV internal diameter) /4 (posterior wall thickness at end systole) $(1+$ (posterior wall thickness at end systole/LV internal diameter). We will be calculating mean arterial pressure using the formula $1 / 3$ systolic $\mathrm{BP}+2 / 3$ diastolic $\mathrm{BP}$ as a surrogate for $\mathrm{LV}$ end-systolic pressure. These diameters will be assessed in parasternal short axis view. ${ }^{16}$

For the right ventricle, measurements include tricuspid annular plane systolic excursion measured by M-mode, systolic annular peak velocities (S') using $\mathrm{TDI}^{15}$ and fractional area of change obtained by ((end-diastolic area end-systolic area)/end-diastolic area). ${ }^{15}$

\section{Diastolic function}

Using PWD, mitral inflow peak early filling (E') and peak atrial filling (A') wave velocities will be measured using the apical four-chamber view, and the $\mathrm{E} / \mathrm{A}$ ratio calculated. Deceleration time will be measured as the time between the peak $\mathrm{E}$ velocity and the point where the velocity returns to zero. TDI derived peak early (E') and peak late (A') diastolic velocities will also be measured at the base of the interventricular septum. All these values will be derived from three consecutive cardiac cycles and 
averaged. The E-wave to $\mathrm{LV}$ E' velocity ratio $(\mathrm{E} / \mathrm{E}$ ') will then be calculated. ${ }^{15}$

\section{Global function}

The myocardial performance index (Tei index) has been used to assess combined LV systolic and diastolic function. It is calculated as isovolumic contraction time plus isovolumic relaxation time divided by ejection time. These timings will be reported separately and also as a collection function in the form of Tei Index value. Each value is recorded over three consecutive beats and averaged out for calculation of the index. It is measured by a PWD positioned at the tip of mitral leaflets in the apical five-chamber view. ${ }^{15}$

\section{Strain analysis}

Using the GE Healthcare Vivid E9 ultrasound system, digital loops with three consecutive cardiac cycles will be acquired from apical two, three and four-chamber view, with frame rate set at more than 60 frames/s(frame rate to heart rate ratio of close to 1 ). Strain analysis, via speckle tracking, will be performed directly on the system for each of the three apical views. The operator will manually identify 2 points on each side of the mitral valve and 1 point at the LV apex. Using the Automated Functional Imaging (AFI) strain technique, the software automatically detects the endocardium and tracks the myocardium during the entire cardiac cycle (refer to figure 2) ${ }^{17}$ Global longitudinal strain and will then be calculated by averaging the 16 segment strains of the LV. Peak global longitudinal strain rate, peak early diastolic filling and peak late diastolic filling strain rates will also be reported. Tracking quality will be assessed by the observer and endocardial tracing will be readjusted or the imaging repeated if the quality is unsatisfactory. ${ }^{17}$ Images with at least four out of six segments deemed to be adequately tracked by the automated system will be selected for analysis. The AFI technique determines aortic valve closure from the threechamber view while the strain analysis begins from the peak of the R-wave on ECG.

Global and regional strain longitudinal will also be calculated from only the four-chamber view. Aortic valve opening and closure will be determined using PWD across the aortic valve with a similar R-R interval on ECG. ${ }^{18} \mathrm{LV}$ endocardium will then be traced and tracking assessed manually. Image will be selected if at least four segments out of the six are considered to be adequately tracked. In this technique, strain analysis will be measured from the beginning of the R-wave on ECG.

\section{Vascular examination}

\section{Carotid intima-media thickness}

The examination will be performed using the Association for European Paediatric Cardiology Working Group on Cardiovascular Prevention guidelines. ${ }^{19}$ The GE Healthcare Vivid E9 ultrasound scanner with a 7-12 MHz linear transducer will be used to acquire the images. The subject will be placed supine in a quiet room with the head

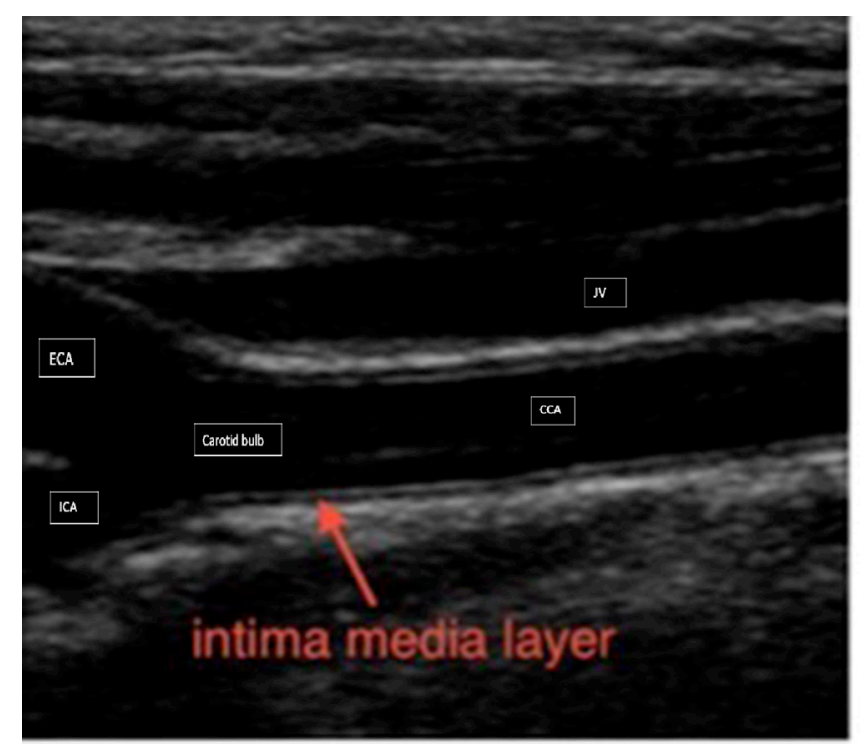

Figure 3 Intima-media thickness of common carotid artery.

extended and turned away from the transducer. Longitudinal images of the common carotid artery both sides will be obtained proximal to the carotid bulb. Minimum $5 \mathrm{~s}$ clip will be recorded and stored for each vessel to allow selection of the most appropriate frame for offline analysis. ${ }^{19}$ cIMT measurements will be taken from the far wall of the common carotid artery, $10 \mathrm{~mm}$ away from the carotid bulb. On the carotid wall, two bright lines separated by a darker area will be identified (refer to figure 3). The IMT software will then be used to delineate 105-110 points on a pixel basis and the average cIMT value (over three consecutive heart cycles) will be calculated for each side.

\section{Pulse wave velocity}

Carotid femoral PWV is calculated by dividing distance travelled by the transit time. ${ }^{20}$ The study participant will be laid supine with the head extended. The right carotid and right femoral artery will be felt manually and PWD will be obtained on each. The distance will be measured by direct distance of common carotid artery to femoral artery pulse multiplied by 0.8 . The transit time will be indirectly obtained by subtracting the time delay between the onset of $\mathrm{R}$ wave and upstroke of common carotid artery and onset of $\mathrm{R}$ wave and upstroke of common femoral artery. ${ }^{20}$ The measurements will be performed offline on DICOM raw data on ECHOPAC software to preserve the frame rate at which the image was acquired (refer to figure 4 ).

\section{Patient and public involvement}

Patients and the public were not involved in the design of this study. However, all study participants will be informed regarding the study findings via email after the study results are published.

\section{Statistical analysis plan}

Data will be entered and analysed using SPSS V.19.0. Data will be presented as mean $\pm \mathrm{SD}$, median $(\mathrm{IQR})$ or 


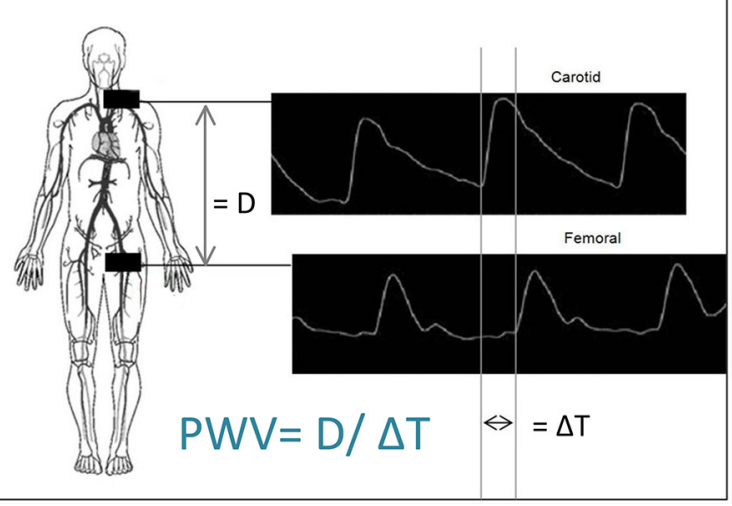

Figure 4 Measurement of pulse wave velocity D-carotidfemoral distance, $\mathrm{T}$-time difference between upstroke on common carotid artery and femoral artery.

percentages as appropriate. Cardiac measurements will be standardised using appropriate $\mathrm{z}$ scores on a software that is freely available on the internet. ${ }^{10}$ Independent t-test or Mann-Whitney $U$ test will be used for testing of continuous variables (based on the assumption of normality). Variables with a $\mathrm{p}<0.1$ or those that are clinically relevant will be used for the multivariable model (one assumptions are fulfilled). Multivariate analysis will control for confounders such as maternal BMI, birth weight, gestational age, child's BMI and heart rate. A $\mathrm{p}<0.05$ will be used to determine statistical significance.

Two independent observers will perform IMT and global longitudinal strain calculations on 20 studies as well to determine interobserver variability. Bland-Altman graphs will be generated to determine the interobserver variability.

\section{ETHICS AND DISSEMINATION}

Findings of this study will be disseminated by means of scientific publications and continuous medical education seminars. The study participants will be informed regarding the study findings through project summaries and sharing of key findings.

\section{STUDY STATUS}

We plan to begin enrolment in July 2018.

Contributors $\mathrm{ZH}$, NM and BSH were involved in the concept, grant writing protocol development and intellectual inputs for this project. DC and BSH provided expert advice and technical training. All authors contributed towards development of this manuscript.

Funding The Aga Khan University Faculty of Health Sciences Grants Research Committee (grant number: 181005PAEDS).

Competing interests None declared.

Patient consent Parental/guardian consent obtained.

Ethics approval Ethical approval has been obtained from the Ethics Review Committee at The Aga Khan University.

Provenance and peer review Not commissioned; externally peer reviewed.

Open access This is an open access article distributed in accordance with the Creative Commons Attribution Non Commercial (CC BY-NC 4.0) license, which permits others to distribute, remix, adapt, build upon this work non-commercially, and license their derivative works on different terms, provided the original work is properly cited, appropriate credit is given, any changes made indicated, and the use is non-commercial. See: http://creativecommons.org/licenses/by-nc/4.0/.

\section{REFERENCES}

1. Sibai B, Dekker G, Kupferminc M. Pre-eclampsia. Lancet 2005;365:785-99.

2. Hutcheon JA, Lisonkova S, Joseph KS. Epidemiology of preeclampsia and the other hypertensive disorders of pregnancy. Best Pract Res Clin Obstet Gynaecol 2011;25:391-403.

3. Woelkers D, Barton J, von Dadelszen P, et al. [71-OR]: The revised 2013 ACOG definitions of hypertensive disorders of pregnancy significantly increase the diagnostic prevalence of preeclampsia. Pregnancy Hypertension: An International Journal of Women's Cardiovascular Health 2015:5:38.

4. Davis EF, Lazdam M, Lewandowski AJ, et al. Cardiovascular risk factors in children and young adults born to preeclamptic pregnancies: a systematic review. Pediatrics 2012;129:e1552-61.

5. Fugelseth D, Ramstad HB, Kvehaugen AS, et al. Myocardial function in offspring 5-8years after pregnancy complicated by preeclampsia. Early Hum Dev 2011;87:531-5.

6. Akcakus M, Altunay L, Yikilmaz A, et al. The relationship between abdominal aortic intima-media thickness and lipid profile in neonates born to mothers with preeclampsia. J Pediatr Endocrinol Metab 2010;23:1143-9.

7. Valensise H, Vasapollo B, Gagliardi G, et al. Early and late preeclampsia: two different maternal hemodynamic states in the latent phase of the disease. Hypertension 2008;52:873-80.

8. Lazdam M, de la Horra A, Diesch J, et al. Unique blood pressure characteristics in mother and offspring after early onset preeclampsia. Hypertension 2012;60:1338-45

9. Uçar T, Tutar E, Yalçinkaya F, et al. Global left-ventricular function by tissue doppler imaging in pediatric dialysis patients. Pediatr Nephrol 2008;23:779-85

10. Boston Children's Hospital, 2015. Z score calculator http://zscore. chboston.org/ (cited 11 Dec 2017).

11. Artang $R$, Migrino $R Q$, Harmann $L$, et al. Left atrial volume measurement with automated border detection by 3-dimensional echocardiography: comparison with Magnetic Resonance Imaging. Cardiovasc Ultrasound 2009;7:16.

12. Lu JC, Ensing GJ, Yu S, et al. 5/6 Area length method for leftventricular ejection-fraction measurement in adults with repaired tetralogy of Fallot: comparison with cardiovascular magnetic resonance. Pediatr Cardiol 2013;34:231-9.

13. Lowes BD, Gill EA, Abraham WT, et al. Effects of carvedilol on left ventricular mass, chamber geometry, and mitral regurgitation in chronic heart failure. Am J Cardiol 1999;83:1201-5.

14. Crispi F, Bijnens B, Figueras F, et al. Fetal growth restriction results in remodeled and less efficient hearts in children. Circulation 2010;121:2427-36

15. Lopez L, Colan SD, Frommelt PC, et al. Recommendations for quantification methods during the performance of a pediatric echocardiogram: a report from the Pediatric Measurements Writing Group of the American Society of Echocardiography Pediatric and Congenital Heart Disease Council. J Am Soc Echocardiogr 2010;23:465-95

16. Colan SD, Borow KM, Neumann A. Left ventricular end-systolic wall stress-velocity of fiber shortening relation: a load-independent index of myocardial contractility. J Am Coll Cardiol 1984;4:715-24.

17. Belghitia $\mathrm{H}$, Brette $\mathrm{S}$, Lafitte $\mathrm{S}$, et al. Automated function imaging: a new operator-independent strain method for assessing left ventricular function. Arch Cardiovasc Dis 2008;101:163-9.

18. Marciniak A, Sutherland GR, Marciniak M, et al. Prediction of postoperative left ventricular systolic function in patients with chronic mitral regurgitation undergoing valve surgery-the role of deformation imaging. Eur J Cardiothorac Surg 2011;40:1131-7.

19. Dalla Pozza R, Ehringer-Schetitska D, Fritsch P, et al. Intima media thickness measurement in children: a statement from the Association for European Paediatric Cardiology (AEPC) working group on cardiovascular prevention endorsed by the association for European paediatric cardiology. Atherosclerosis 2015;238:380-7.

20. Van Bortel LM, Laurent S, Boutouyrie P, et al. Expert consensus document on the measurement of aortic stiffness in daily practice using carotid-femoral pulse wave velocity. $J$ Hypertens 2012;30:445-8. 